\title{
Study of the Lateral Boundary Condition Temporal Resolution Problem and a Proposed Solution by Means of Boundary Error Restarts
}

\author{
Piet Termonia, Alex Deckmyn, And Rafiq Hamdi \\ Royal Meteorological Institute, Brussels, Belgium
}

(Manuscript received 3 February 2009, in final form 6 April 2009)

\begin{abstract}
To properly utilize coupled limited-area models (LAMs), the time scales of the cross-boundary fluxes in the available lateral boundary data must be assessed. In current operational practice, the update frequencies of these data are usually determined by common sense guesswork and by technical constraints. This paper quantifies the required temporal resolution of the lateral boundary conditions. For a mesoscale LAM it is concluded that in standard forecast cases, coupling updates of about $3 \mathrm{~h}$ are sufficient. However, in rare cases of severe storms, this can lead to errors in the coupling data of about $10 \mathrm{hPa}$. To avoid such errors, it is found that one should update the coupling fields with the period given by the time step of the model that provides the coupling data. However, in most existing operational applications this is not feasible. For those cases, it is shown that the forecast can be substantially improved by restarting the model run at a forecast range when the storm has entered the domain. The proper restart time can be detected in an operational suite by an existing strategy of monitoring the coupling update frequency. Additionally, it is argued that the forecast should then be initialized by a scale-selective digital filter.
\end{abstract}

\section{Introduction}

Limited-area models (LAMs) are ubiquitously used nowadays in numerical weather prediction, climate modeling, and air quality applications. Because of the limitations of their domain size, they can be run at higher resolutions than global models for comparable computational costs and, as a consequence, they are believed to have more skill in simulating extreme weather situations that are of primary societal interest, for instance, severe storms.

To run numerical weather prediction (NWP) models on limited areas, the lateral boundary conditions (LBCs) have to be specified, but the existing numerical techniques used to impose them in operational models still exhibit, as Warner et al. (1997) properly address, a number of potentially serious limitations. A particular problem discussed in that paper is the one of the $L B C$ temporal resolution. Warner et al. state that, "the time scales of the cross-boundary fluxes must be assessed, and the temporal resolution of the LBCs should be defined accordingly."

Corresponding author address: P. Termonia, Royal Meteorological Institute of Belgium, Ringlaan 3, B-1180 Brussels, Belgium. E-mail: piet.termonia@oma.be
The LBC temporal resolution problem as it will be specifically studied in the present paper, is, in its most general form, formulated as follows. The LAM needs the values of the dynamical fields at its lateral boundaries and these data are constructed from the model output of a coupling model. This is usually a global model where no LBC problem is present, but which is run with a coarser resolution. As a consequence, the time step of this coupling model is larger than the one required by the dynamical scheme of the LAM. Moreover, in order to keep the input/output (I/O) of the coupling models within reasonable limits, these data are usually produced with time intervals that are multiples of hours. They are then temporally interpolated to get data with the temporal resolution of the time-stepping scheme of the LAM.

For instance, in European operational applications, the LBC data are often created with a coupling update interval of $3 \mathrm{~h}$ from the model output of global models that are run with time steps of typically $15 \mathrm{~min}$. Mesoscale LAMs often run with time steps closer to about 5 min or even smaller. The actual lateral boundary data are created by interpolating the 3-h coupling data to create time series with 5-min resolution. So although the LAM effectively uses 5-min data, the used time series 
will not contain any physically meaningful information on time scales smaller than $3 \mathrm{~h}$.

In fact, this issue has been the source of a problem studied by Termonia (2003, hereinafter T03) in a mesoscale forecast of the famous European Lothar storm in 1999 (see Wernli et al. 2002). The use of a coupling update interval of $3 \mathrm{~h}$ had a detrimental effect on the forecast quality of this storm, reducing the depth of the low by $7-8 \mathrm{hPa}$ compared to the depth of the storm in the forecast of the coupling model. Indeed, as the analysis in the present paper will show, variations in pressure of about this magnitude can occur on a time scale smaller than $3 \mathrm{~h}$, and the corresponding information is then completely lost or corrupted by the current practice of interpolating 3-h coupling interval updates.

Another aspect raised in Warner et al. (1997) is the current state of the mathematical formulations of the LBCs, which is still far from accurate. Usually, LAMs rely on engineering solutions such as, for instance, the flow relaxation scheme of Davies (1976). Current evolutions in numerical weather prediction (NWP) to kilometer-scale modeling in Europe have revived the interest in alternative, more accurate LBC formulations (see, e.g., McDonald 2005; McDonald 2006; Termonia and Voitus 2008; Voitus et al. 2009). The temporal frequencies involved at these scales are expected to be higher than the ones studied up to now in the literature. This reinforces the need for a more systematic approach of this LBC temporal resolution problem.

Two guidelines put forth by Warner et al. (1997) are specifically relevant for the present paper. A first guideline is to "utilize a lateral-boundary buffer zone," that is, to move the lateral boundaries sufficiently far away from the area of meteorological interest within the computational domain such that lateral boundary errors do not reach this area during the entire forecast range. By doing so, one hopes that the features reaching it have all been created within the domain of the LAM. This has been conventional wisdom since the introduction of LAMs into operational applications and can be quantified by various methods [see, e.g., the standard paper of Baumhefner and Perkey (1982) and more recently Vannitsem and Chomé (2005)]. A second guideline in Warner et al. (1997) is to "avoid strong forcing at the lateral boundaries." In that paper this guideline pertains specifically to strong orographic forcing, and it is recommended to select the lateral boundaries such that they do not pass regions of steep orography.

In contrast to these two guidelines, the literature on the $\mathrm{LBC}$ temporal resolution problem is very thin. But a particular case is the operational forecast of the famous 1999 Lothar storm studied in T03. The occurrence of a storm of this strength is very rare, but ironically, it is, for many national meteorological services, one of the main reasons to rely on LAMs.

A potential solution to this problem has been suggested in a paper subsequent to T03, namely, Termonia (2004, hereinafter T04). Termonia showed that such cases can actually be anticipated before the run of the LAM is carried out, by a strategy of monitoring the coupling update frequency. This consists of applying a temporal digital high-pass filter on the surface pressure field during the run of the coupling model. If the cutoff time of this digital filter is chosen to be the abovementioned coupling update frequency $(3 \mathrm{~h}$ in the example), this high-pass filtered surface pressure (HFSP) field will provide a good estimate of the high-frequency part of the signal that is lost after the interpolation to the time step of the LAM. And, as will be shown in the present paper, this information can be used to make an estimate of the potential error introduced by the interpolation of the coupling data.

In T04 it was also proposed to approximate this HFSP by a second-order recursive filter, such that the filter can be applied during the forecast of the coupling model. This can be easily implemented within an operational suite and the resulting HFSP can be supplied as part of the coupling data for the LAM. In T04 several strategies for improving the LBC temporal resolution problem of fast-propagating storms were proposed. One of them is to restart the forecast at times when the absolute value HFSP exceeds a predefined critical value. The correct detection of the restart time will guarantee that the storm is inside the domain at the time of the restart. After the restart, the storm will continue to propagate further within the domain without having been present on the boundaries. As will be shown in the present paper, this can be used to substantially improve the forecast.

In T03 and T04, the issue of the choice of an adequate coupling update interval to guarantee a specific quality of the interpolated coupling data, has not been addressed, and this is, in today's operational practices, still based too much on common sense guesswork. The aim of the present paper is to provide a quantitative approach for this problem.

The Lothar storm has been the most extreme one encountered in the operational suites of the Aire Limitée Adaptation Dynamique Développement International (ALADIN) LAM (see ALADIN International Team 1997), so the required temporal resolution of the LBCs for this case must also be adequate in less extreme cases. Within the studied framework of the ALADIN model, the present paper shows that this turns out to be the time step of the coupling model, that is, about $15-20 \mathrm{~min}$. In many operational applications it is technically not 
feasible to provide coupling data with such high coupling update frequencies.

The present paper proposes a solution by the use of a nesting strategy that employs the HFSP to detect boundary errors, and when they occur, it proposes to carry out restarts of the forecast, henceforth referred to as boundary error restarts (BERs). Instead of relying on the LBC data that have been corrupted by the temperal resolution problem on the lateral boundaries, such a BER uses the uncorrupted data of the coupling model when the storm is already inside the domain of the LAM. This proposal will be elaborated upon in detail and some crucial subtleties concerning the initialization will be addressed. It will then be shown that the forecasted strength of the storm is substantially improved after the restart time by the restarted model run. It will also be concluded that, based on about $2 \mathrm{yr}$ of operational HFSP data computed within the global Action de Recherche Petite Echelle Grande Echelle (ARPEGE) model of Météo-France, BERs are needed only a few times per year to correct the most extreme storms. So the operational suites can still be run with 3-h coupling updates and they will be restarted only very rarely, to produce better estimates of the strength of the storm.

Based on the studied data, it will be explicitly shown that with this procedure, the incoming extreme storm will always be inside the domain at the restart. Such a storm is a strong forcing, but a dynamical one instead of an orographic one. Hence, this approach provides a solution for the two guidelines of Warner et al. (1997) of using a buffer zone and the avoidance of the strong forcings at the boundaries mentioned above.

This paper is organized as follows. Section 2 will provide an analysis of the temporal resolution problem in the case of the above-mentioned Lothar storm, and will provide an estimate of the required coupling update frequency. Section 3 will elaborate upon the idea of the boundary error restarts that has already been briefly mentioned in T04. A final discussion will be presented in section 4 .

\section{The lateral boundary condition temporal resolution problem}

In this section the required temporal resolution for an adequate coupling for the Lothar storm forecast studied in T03 will be derived. The model used for the present study is the ALADIN model developed by the ALADIN International Team (1997). The same setup of this model has been used for the studies in T03 and T04, and a more detailed description of the model data can be found therein. This scheme is running operationally at the Royal Meteorological Institute (RMI) of Belgium. In
T04 it is argued that the efficiency of the coupling update frequency has to be examined in the coupling model. The analysis in this section was thus carried out using the operational version of the ALADIN model running at Météo-France, which is the model providing the coupling data for the operational ALADIN version at the RMI.

The prognostic variable of the ALADIN model giving the best signature of a storm is the logarithm of the surface pressure: $\Pi=\ln P_{s}$. However, for the sake of this discussion, the method is applied to the surface pressure $P_{s}$ here, because this allows us to identify the required coupling update frequency in a more physically intuitive manner. Exactly the same analysis has been carried out for $\Pi$ and this led to the same conclusions.

In contrast, in the ALADIN model used in T04, the high-pass digital filter has been applied to its model variable $\Pi$ instead of $P_{s}$. This can also be seen as a kind of filtering of the surface pressure, albeit indirectly, and this will, conveniently, also be referred to as the HFSP henceforth. ${ }^{1}$

The maximum of the absolute value of this HFSP has been sought within the dataset that was used in T04 of the ALADIN Météo-France model output during all the 0000 UTC based forecasts during December 1999. Not surprisingly, the maximum value was found in the mean sea level pressure (mslp) of the Lothar storm in the forecast on 26 December 1999 based on the 0000 UTC analysis. Figure 1 shows the mslp of this storm. The selected point is indicated in Fig. 1 at the time when the eye of the storm passed through it.

Figure 2 shows the discrete time series of $P_{n}=P_{s}\left(t_{n}\right)$, with $n=0, \ldots, N-1$, at this point during the forecast. The eye of the storm passed through this point at 0755 UTC. Assuming that the eye lays within the coupling zone of a LAM, the required temporal resolution will be assessed to adequately represent the signal of $P_{s}$.

From a signal processing point of view, the procedure described in the introduction consists of two steps. The first is a resampling of the time series with a lower temporal resolution defined by a time interval $T$. This is a multiple of the time step of the coupling model. Thus, from this series, each time $\tau_{\alpha}=\alpha T$, with $\alpha$ being an integer, the coupling data of the LAM are updated from the output of the coupling model. The second step is recreating a time series of the higher temporal resolution corresponding to the time step of the LAM, by means of an interpolation. The values at times $s_{I}=I \Delta t$, with $I$ an integer and with $\Delta t$ the time step of the LAM,

\footnotetext{
${ }^{1}$ In fact, whether HFSP refers to $P_{s}$ or $\ln P_{s}$ will be always clear from the context.
} 


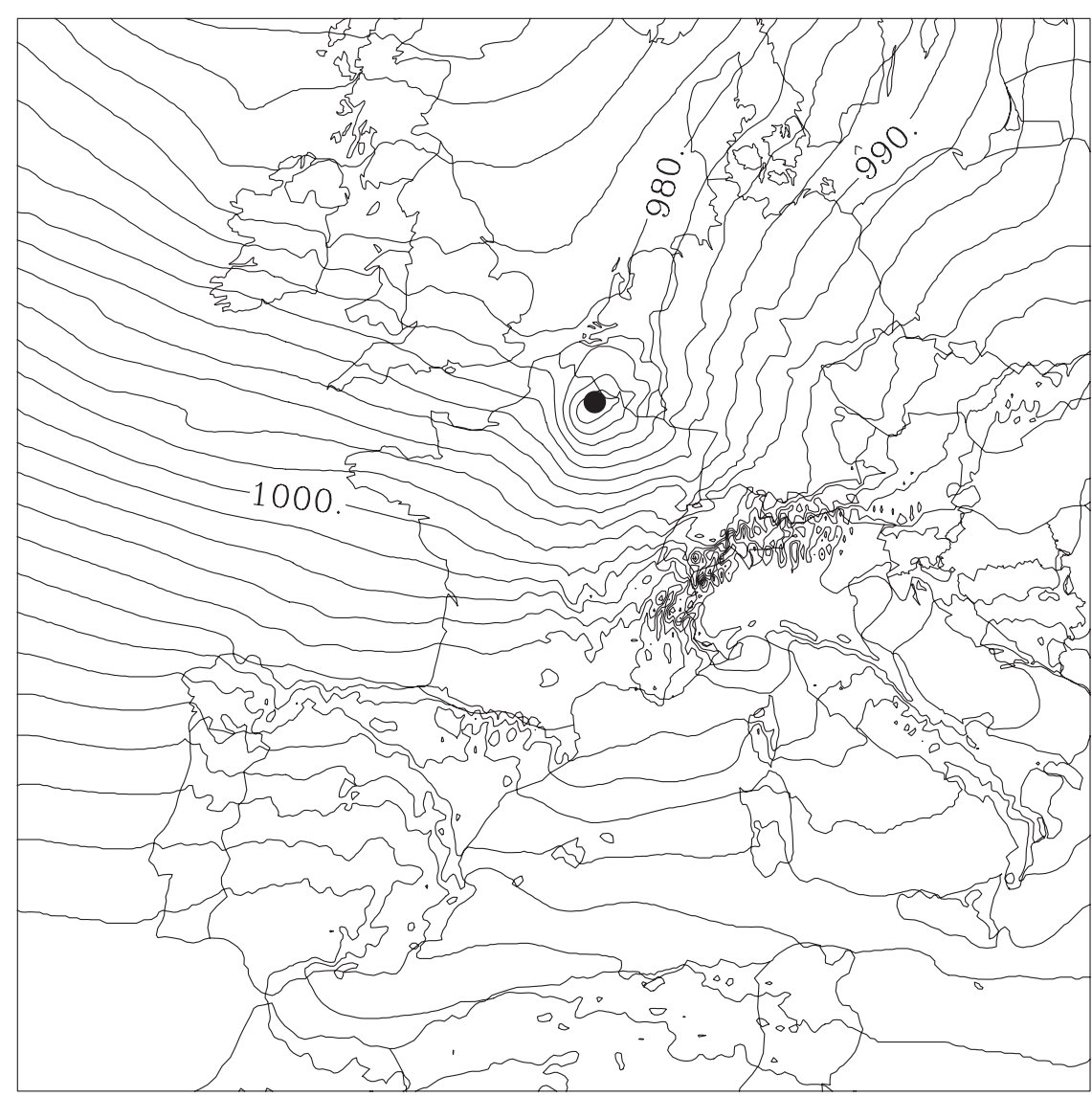

FIG. 1. ALADIN mslp at 0755 UTC 26 Dec 1999. The selected point is indicated by the black dot at $49^{\circ} 57^{\prime} 50^{\prime \prime} \mathrm{N}, 4^{\circ} 2^{\prime} 16^{\prime \prime} \mathrm{E}$ in the eye of the storm.

are then obtained by an interpolation such as, for instance, the linear interpolation $\mathcal{L}$,

$$
\mathcal{L}_{T, \tau} P(t)=\frac{T+\tau-t}{T} P(\tau)+\frac{t-\tau}{T} P(\tau+T)
$$

between time $\tau$ and time $\tau+T$, where $\tau$ is the first time instant of the interpolation interval.

Note that if the times series were taken in a point slightly later on the storm track in Fig. 1, that is, the boundary lay farther to the east, the same type of series would be found as in Fig. 2, but the minimum of $P_{s}$ would have been found later than 0755 UTC. This would shift the times of the coupling updates $\tau_{\alpha}$ relatively with respect to the minimum of $P_{s}$. This uncertainty of the position of the lateral boundaries and the times of the coupling updates with respect to the time series, should be taken into account when making an estimate of the potential error that could be made by the interpolation.

The coupling update interval $T$ should be chosen small enough to keep the discrepancy between the interpolation and the original time series of the coupling model below a predefined acceptable maximum. This is achieved by considering the maximum

$$
E^{\max }(T)=\max _{\tau_{n} \in[0 \mathrm{~h}, 48 \mathrm{~h}-T]}\left\{\max _{t_{m} \in\left[\tau_{n}, \tau_{n}+T\right]}\left[\left|P_{m}-\mathcal{L}_{T, \tau_{n}} P\left(t_{m}\right)\right|\right]\right\},
$$

with $P_{m}$ a shorthand notation for $P\left(t_{m}\right)$, and $\tau_{m}$ and $t_{m}$ the $m$ th time level of the coupling model. By choosing $T$ such that $E^{\max }(T)$ is less than or equal to the predetermined accepted maximum, it is ensured that this maximum will not be exceeded. For example, the maximal difference for $T=3 \mathrm{~h}$ in (2) can be seen in Fig. 2. It occurs at 0755 UTC for the interpolation between $\tau=$ 0615 UTC and $\tau+T=0915$ UTC. The difference itself yields $E^{\max }(3 \mathrm{~h})=11.5 \mathrm{hPa}$. So this analysis shows that, as a worst case, using coupling data with a coupling update frequency of $3 \mathrm{~h}$ may potentially cause errors as large as this. It is also obvious that, for passing depressions as the one in Fig. 2, any interpolation over a too long time interval will always make the interpolated low less deep than the original one. 


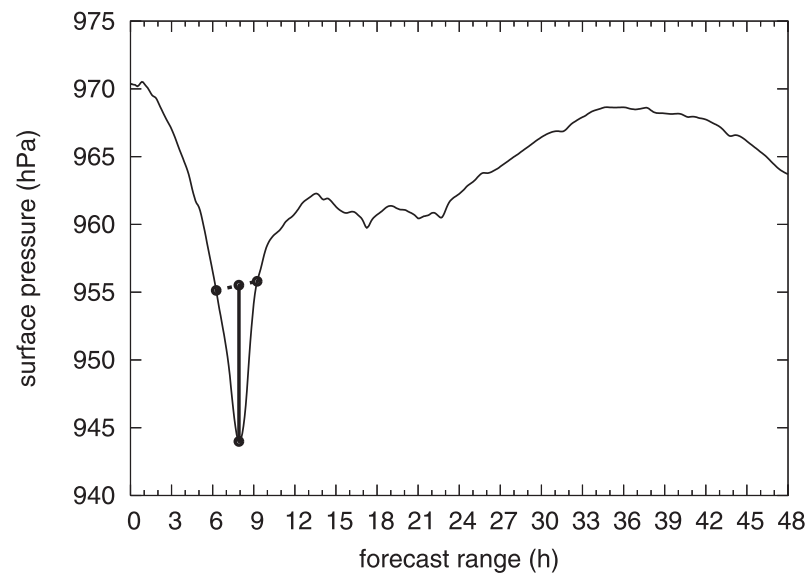

FIG. 2. Time series of $P_{s}$ at the point on $49^{\circ} 57^{\prime} 50^{\prime \prime} \mathrm{N}, 4^{\circ} 2^{\prime} 16^{\prime \prime} \mathrm{E}$ indicated in Fig. 1. For interpolation time interval $T=3 \mathrm{~h}$, the greatest difference between the interpolation and the value of the times series (solid thick line) occurs at 0755 UTC for the interpolation between $\tau=0615$ UTC and $\tau+T=0915$ UTC (dashed thick line). The difference between the minimum and the linear interpolation is $11.5 \mathrm{hPa}$.

If the original time series $P_{n}$ contains frequencies higher than the Nyquist frequency $f_{N}=1 /(2 T)$, then, after the resampling, they will be aliased. So, actually aliased data are then interpolated in $\mathcal{L}_{T, \tau} p\left(S_{I}\right)$. The spectral content in the higher frequencies can be estimated by the spectral decomposition:

$$
\tilde{P}_{k}=\frac{1}{N} \sum_{n=0}^{N-1} P_{n} e^{-2 \pi i f_{k} t_{n}}
$$

for $k=-N / 2, \ldots, N / 2$ and where $f_{k}=k /(N \Delta t)$ is the frequency corresponding to the mode $k$.

To take a fast Fourier transform (FFT) and its inverse [see Eq. (5) below], the time series must be made periodic. Following Errico (1985), this can be done by removing a trend before taking the FFT:

$$
P_{n}^{\text {detrended }}=P_{n}-\frac{n}{N-1}\left(P_{n}-P_{0}\right)
$$

So, Eq. (3) is computed using $P_{n}^{\text {detrended }}$ instead of $P_{n}$. The label "detrended" will be dropped henceforth. This does not influence the difference between the linear interpolation and the time series since the linear interpolation of a linear trend is the trend itself.

From the form of the inverse FFT of Eq. (3),

$$
P_{n}=\sum_{k=0}^{N-1} \tilde{P}_{k} e^{2 \pi i f_{k} t_{n}}
$$

it follows that the discrepancy between the interpolated and original time series, $\delta_{T, \tau} P_{n}=P_{n}-\mathcal{L}_{T, \tau} P\left(t_{n}\right)$, can be treated by considering the discrepancy of each mode $\exp \left(2 \pi i f_{k} t_{n}\right)$ separately. For modes with frequencies $|f| \leq 1 /(2 T)$, this difference is always largest in the middle of the time interval $t_{0}+T / 2$ (see Fig. 1 in T04). In that case,

$$
\left.\mathcal{L}_{T, t_{0}} e^{2 \pi f t}\right|_{t=t_{0}+T / 2}=\cos (\pi f T) e^{2 \pi f\left(t_{0}+T / 2\right)} .
$$

The information lost by the interpolation can thus be quantified by $1-\cos (\pi f T)$.

The information in the frequencies $|f|>1 /(2 T)$ is completely lost. So the net information loss of the resampling and the interpolation can be quantified by means of a high-pass filter:

$$
H_{T}(f)=\left\{\begin{array}{lll}
1-\cos (\pi f T) & \text { if } & |f| \leq \frac{1}{2 T} \\
1 & \text { if } & |f|>\frac{1}{2 T}
\end{array}\right.
$$

Besides taking account of the aliasing, this approach of the filtering with (7) also has the advantage that it can be implemented as a recursive digital filter. In T04 this has been approximated ${ }^{2}$ by a second-order recursive Butterworth filter applied on the logarithmic surface pressure. The advantage of this approximation is that it can be applied in any grid point during a run of the model that provides the coupling data for the LAM. In a small zone near the lateral boundaries of the LAM, the resulting field (i.e., the HFSP) thus provides an estimate of the maximal potential loss of information made by the temporal interpolation between any possible coupling update times $\tau$ and $\tau+T$. This information can then be used in operational suites for monitoring the coupling update frequency.

The approach of the present paper is different from that in T04; here, the analysis of the time series is restricted to some specific singular grid points of the domain (e.g., the time series in Fig. 2), which allows for the computation of the information loss exactly by means of (7). Also, it is impossible to operationally compute $E^{\text {max }}$ for all grid points within a model run, since to compute $E^{\max }$ with (2), it would be necessary to store all the values of the logarithmic surface pressure at all time steps of the coupling model and the computation could only be carried out on this huge dataset after the model run is finished.

From (5), it follows that, for any chosen time with index $n$, the absolute value of the filtered signal taken in the time domain has an upper bound $\Lambda$ given by

\footnotetext{
${ }^{2}$ See Fig. 2 in T04, comparing the response function of the recursive filter with $H_{T}$ in Eq. (7) of the present paper.
} 


$$
\left|\left(h_{T} * P\right)_{n}\right| \leq \sum_{k=0}^{N-1} H_{T}\left(f_{k}\right)\left|\tilde{P}_{k}\right|=\Lambda(T),
$$

where the asterisk denotes the convolution of the signal with the response function of the high-pass filter in the time domain. In T04 the digital filter had to be applied to all of the grid points during the entire model run to make an estimation of the information loss for a given specific coupling update interval $T$. To estimate the information loss with this method for another value of $T$, one has to rerun the model. In contrast, if the analysis is restricted to the time series of the surface pressure in a specific selected grid point, in particular the one in Fig. 2, then $\Lambda$ can be easily obtained for any given $T$ without having to rerun the model and, thus, the function $\Lambda(T)$ can be constructed. The quantity $\Lambda(T)$ is a measure of the maximally possible information loss between the interpolation and the original value.

Note that, by construction, $\Lambda(T)$ is a monotonically increasing function. So the maximal possible information loss decreases by decreasing the coupling update interval $T$ of the interpolation. The LAM user can decide what the acceptable maximum of the potential loss of the data transfer will be and the needed coupling time interval is then given by $T(\Lambda)$.

Figure 3 shows $T(\Lambda)$ and $T\left(E^{\max }\right)$ for the time series of the mslp of the Lothar storm run on 26 December 1999 and also, for comparison, $T(\Lambda)$ for a run of an anticyclonic calm weather type on 18 December 1999. If the LAM user requires that the temporal error never exceeds $1 \mathrm{hPa}$ in severe storm cases, Fig. 3 shows that the user should employ a coupling-update frequency of about 20 min which is, as discussed in the introduction, roughly the time step of the currently used global models. It can also be seen in Fig. 3 that, for the ordinary case of 18 December, $T=3 \mathrm{~h}$ is sufficient.

For completeness, the same analysis has been carried out for the temperature $(T)$ and the wind components $(u, v)$. The same high-pass recursive filter as the one used for the HFSP has also been applied on the variables $T, u$, and $v$, during the same model runs that were studied in T04. Within these constructed data, for each of these three variables, the grid point and the model run have been identified in which the maximum of the absolute value of these filtered fields occurred. Figure 4 shows the model run time series of these variable in the selected grid points, as well as their corresponding diagrams for $E^{\max }$ and $\Lambda$. It can be seen from Fig. 4 that to guarantee that the error in the interpolation of the temperature does not exceed $1 \mathrm{~K}$, updating with the time step of the coupling model is a priori necessary. It can also be seen that for the zonal wind it is even impossible to guarantee

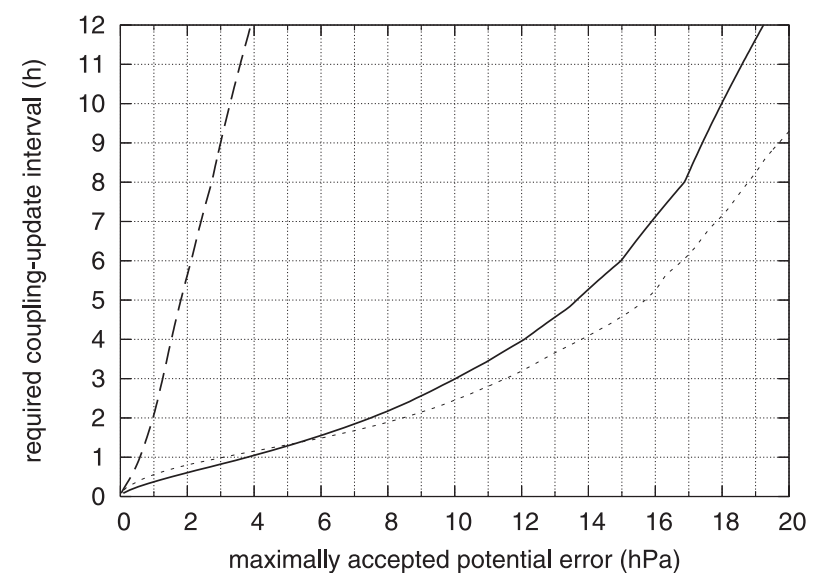

FIG. 3. The required coupling time interval (h) to guarantee an upper bound for a maximal potential error $T\left(E^{\max }\right)$ (dotted line) and the information loss $T(\Lambda)$ (solid line), for $P_{s}$, for the 48-h runs based on the 0000 UTC 26 Dec 1999 analyses, and, for comparison, $T(\Lambda)$ on 18 Dec 1999 (dashed line).

maximum errors of under $1 \mathrm{~m} \mathrm{~s}^{-1}$. However, the wind components may have a strong gravity wave component. Since the Davies scheme (Davies 1976) is applied to filter these gravity waves, they are irrelevant for the current analysis. The meridional wind in the forecast of 5 December 1999 is more interesting from this point of view. It contains a slow component that changes direction at 27-h forecast range. Some fluctuations of an order of magnitude smaller than this component are superposed on this component. In that case the signal represents much more of the rotational part of the dynamics. Here, too, coupling with the time interval of the coupling model is necessary to guarantee maximal errors of the order of a few meters per second.

In conclusion, the only choice of updates that guarantees a sufficient data transfer is the time step of the coupling model. As mentioned in the introduction, this is often impossible in practice. The coupling model may be running at another institution and the data have to be transferred through some telecom system. In the case where the global model is running on the same computer as the LAM, coupling updates with a time interval of the time step of the coupling model should be considered.

The strategy proposed here is first to establish a baseline coupling update frequency. For the present setup, $3 \mathrm{~h}$ is sufficient. Second, the maximum of the absolute value of the HFSP in a frame (e.g., see Fig. 5 below) near the lateral boundaries is considered. In the case of large boundary errors, the user of the LAM can then either (i) perform a restart of the forecast to have the storm inside the domain (as was already described in the introduction) or (ii) download more coupling data in 

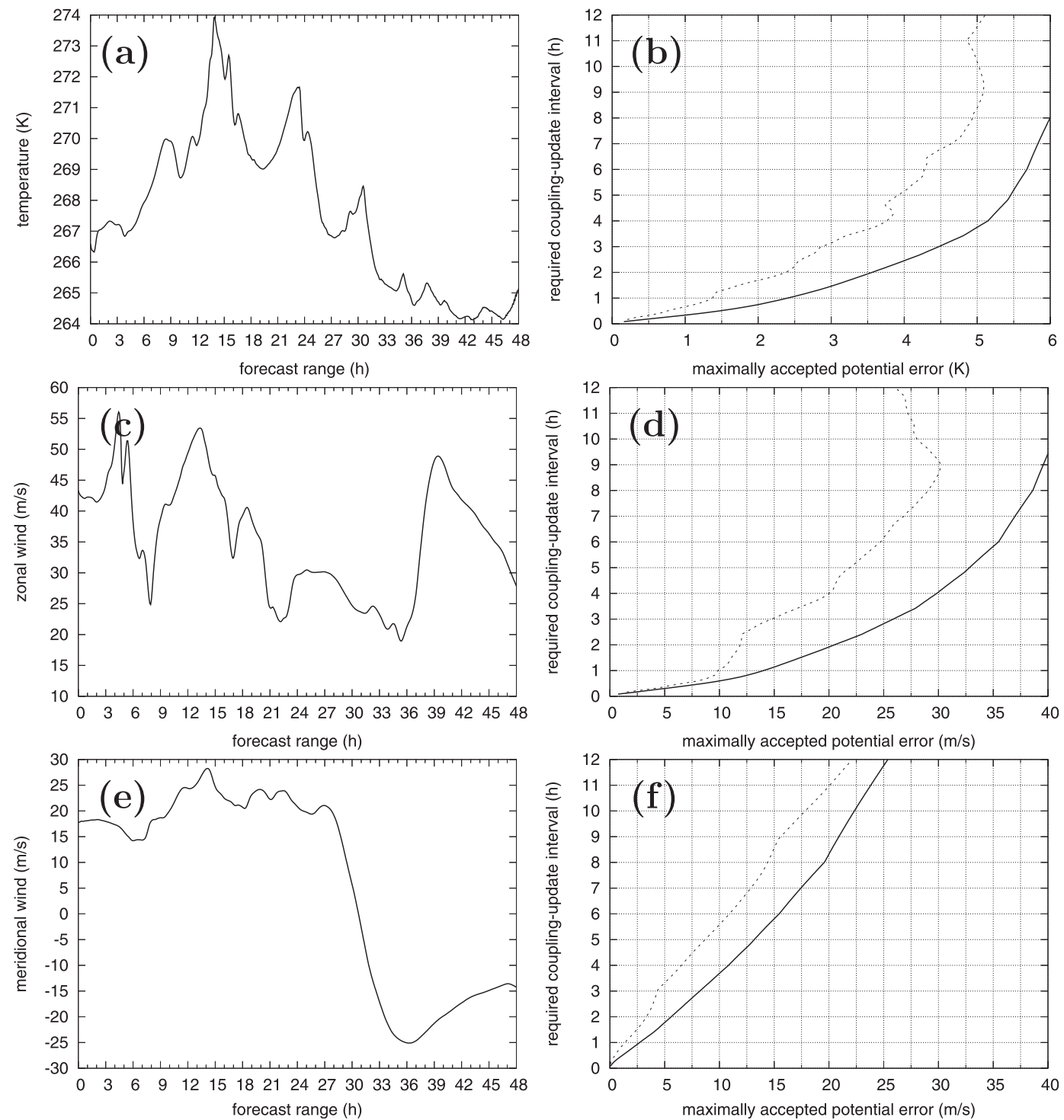

FIG. 4. Time series of (a) temperature and (b) its guaranteeing update interval for the 0000 UTC 26 Dec 1999 run at grid point $47^{\circ} 42^{\prime} 56^{\prime \prime} \mathrm{N}, 15^{\circ} 35^{\prime} 53^{\prime \prime} \mathrm{E}$ at level 27. (c) The zonal wind component and (d) its guaranteeing update interval for the 0000 UTC 26 Dec 1999 run at grid point $49^{\circ} 42^{\prime} 29^{\prime \prime} \mathrm{N}, 4^{\circ} 1^{\prime} 50^{\prime \prime} \mathrm{E}$ at level 14 . (e) The meridional component and (f) its guaranteeing update interval for the 0000 UTC 5 Dec 1999 run at grid point $45^{\circ} 10^{\prime} 1^{\prime \prime} \mathrm{N}, 16^{\circ} 35^{\prime} 46^{\prime \prime} \mathrm{E}$ at level 14. The same conventions as in Fig. 3 are used.

the interval where the threshold value was exceeded to update the boundary conditions with the frequency given by the time step of the coupling model.

The strategy of carrying out a boundary error restart (BER) when an error is detected in the interpolation of the coupling data is most in agreement with the standard LAM coupling philosophy advocated by Warner et al. (1997). Indeed, then we are sure to run the model with the feature of interest inside the domain from the (re)start and a strong forcing in the lateral boundary coupling has been avoided. The disadvantage, on the other hand, is that, in this manner, the small-scale details already obtained by the preceding part of the LAM forecast are lost.

In the remaining part of this paper, a nesting procedure will be proposed based on BERs. Additionally, the following illustrates how this approach works in practice and we will show how the restarts improve the strengths of the most severe storms.

\section{Boundary error restarts}

All ALADIN LAMs are coupled to the ARPEGE global model of Météo-France, either directly or in a 
double-nesting procedure. ${ }^{3} \mathrm{~A}$ decision was made to implement the computation of the HFSP field within ARPEGE operationally, with a second-order Butterworth filter with critical frequency $\omega_{c}=0.9 \pi / \Delta t$ with $\Delta t=3 \mathrm{~h}$, exactly as proposed in T04. This field is passed via the coupling data files to the nested and double-nested ALADIN models. Thus, this HFSP is present in the coupling data beginning on 21 February 2006. It is studied here until 21 June 2008.

In T04 the HFSP is spectrally truncated in space ${ }^{4}$ to avoid the detection of small-scale surface pressure tendencies that are the result of the orography. Here, the truncation is up to modes of maximum wavelength $l_{\max }=171 \mathrm{~km}$. Figure 5 shows the domain on which the coupling data are provided. The fields on this domain have a spatial resolution of $9.5 \mathrm{~km}$. The Davies relaxation zone of the coupled ALADIN LAM is indicated by the dashed lines. The coupled ALADIN model has a grid increment of $7 \mathrm{~km}$. The HFSP is considered within a frame, of 14 grid points wide, indicated by the solid lines. It can be seen that this HFSP frame covers the Davies relaxation entirely. There is a difference in the rotation of both domains due to a difference in the coordinate projections between the two models.

Figure 6 shows the maxima within this frame of the absolute value of the HFSP, for the period 21 February 2006-21 June 2008. The procedure is used to perform a BER when, for a particular coupling time, this maximum exceeds a predefined critical value, $\mu_{c}$. Henceforth, the exceedance will be called the boundary sampling error detection, and its corresponding time the boundary sampling error detection time. If one wants to select roughly the 10 strongest storms in this 2-yr dataset, one needs to perform BERs when the maximum of the HFSP fields exceeds the value of

$$
\mu_{c}=0.003
$$

This is less than the value of 0.004 proposed in T04. However, the two values cannot be exactly compared because the choices of the domain and the spectral truncation were different in that paper. For the current choice, we found eight boundary sampling error detection times in about $2 \mathrm{yr}$, which is relatively rare.

The boundary sampling errors detected in this dataset are summarized in Table 1. Figures 7 and 8 show the mslp at all these boundary sampling error detection times. On

\footnotetext{
${ }^{3}$ For instance, some LAMs are coupled to another LAM of coarser resolution, which in turn is coupled to the global ARPEGE model.

${ }^{4}$ ALADIN is a spectral limited area model with a structure similar to that in Haugen and Machenhauer (1993).
}

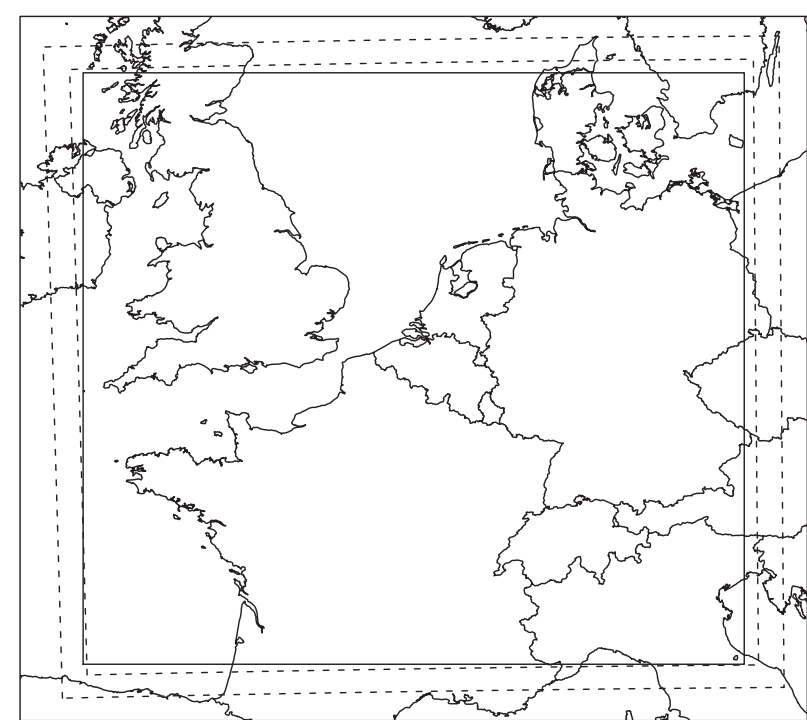

FIG. 5. The frame of the coupling model for the monitoring of the coupling update frequency (solid line) covers the frame of the Davies zone of the LAM (dashed line) but is slightly larger, and the two are relatively rotated due to the different coordinate projections of the coupling and the LAM.

24 November 2006 (Figs. 7a and 7b) and 31 December 2006 (Figs. 8a and 8b), there were two subsequent boundary sampling error detections. It can be seen in Figs. 7 and 8 that these corresponded to the same storm.

There are three different types of boundary sampling error detections, corresponding to whether the storm is (i) incoming, (ii) outgoing, or (iii) tangent to the boundary. Figures $7 \mathrm{a}$ and $7 \mathrm{~b}$ show the mslp at the boundary sampling error detection times for an incoming storm in the 0000 UTC 24 November 2006 run. There was a boundary sampling error detected at the 24- and $27-\mathrm{h}$ forecast ranges. At $27 \mathrm{~h}$ the storm was already mostly inside the domain. The high maximal HFSP value of 0.0058 was caused by the tail of the storm in the western part of the HFSP frame. The incoming case on 25 November 2006 shown in Fig. 7c is actually the same storm but appears in the forecast that is carried out $24 \mathrm{~h}$ later. This is to be compared to Fig. 7b. The second incoming case was found in the 0000 UTC 8 December 2007 run and is shown in Fig. 8d. The outgoing case in the 0000 UTC 18 January 2007 forecast in Fig. 8c is actually the famous Kyrill storm. The boundary sampling error was detected when the storm left the domain. There was another case on 1 December 2006, where a storm entered the northeast corner of the domain and left it at the northern boundary $3 \mathrm{~h}$ later (see Fig. $7 \mathrm{~d}$ ). This can be seen as an incoming case with respect to the western boundary but at the same time as an outgoing case with respect to the northern boundary. Figures $8 \mathrm{a}$ and $8 \mathrm{~b}$ show the tangent case in the 0000 UTC 31 December 


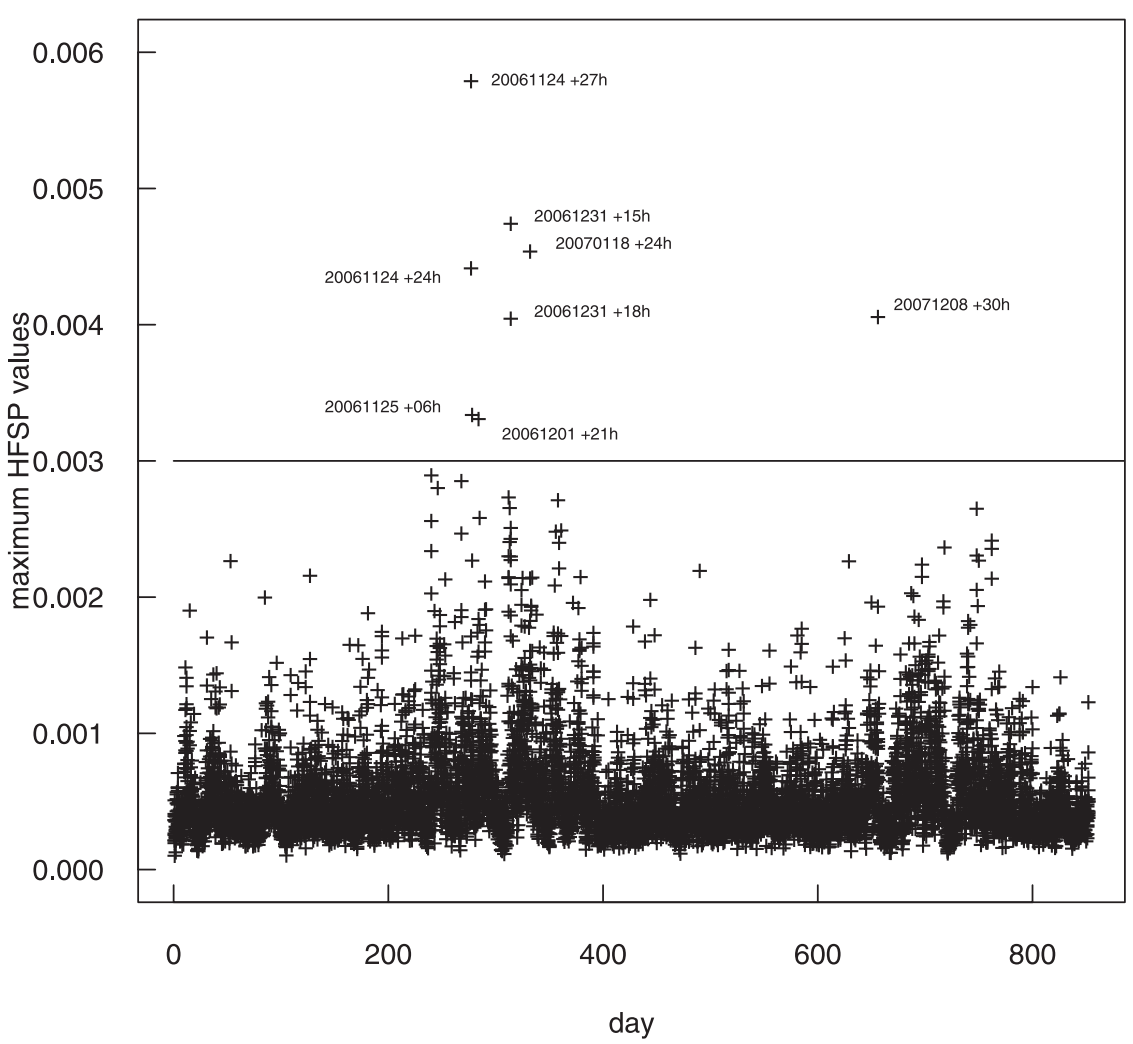

FIG. 6. The maximum of the HFSP values as computed within the ARPEGE model during 21 Feb 2006-30 Jun 2008, within a 14-gridpoint-wide zone covering the Davies relaxation zone of the LAM. The horizontal line is the chosen threshold value of 0.003 .

2006 run. Indeed, the storm is following the northern boundary of the LAM between the 15- and 18-h forecast ranges. Afterward, it leaves the domain to the north. For the choice of $\mu_{c}=0.003$, there were six storms detected by the boundary sampling error detections in a period of $2 \mathrm{yr}$; none of them being a false detection.

For the incoming storms in Table 1, it was found that the use of the corrupted coupling data always led to a weakening of their strength in the LAM forecast compared to the original one in the coupling model, in precisely the same manner as the depth of the Lothar storm was reduced by about $8 \mathrm{hPa}$ relative to the one in the coupling model in T03 (see their Fig. 3). It will be illustrated below, for the cases in Table 1, that carrying out a restart when a boundary sampling error is detected substantially improves the strength of such incoming storms.

When a boundary sampling error is detected, a restart is carried out $3 \mathrm{~h}$ later:

$$
\begin{aligned}
\text { BER time }= & \text { boundary sampling error } \\
& \text { detection time }+3 \mathrm{~h} .
\end{aligned}
$$

In the cases of the two subsequent boundary sampling error detections, this is carried out, in the present paper, for the first one only. The boundary sampling error detection time is added for all the cases to Table 1. Since this represents only a few restarts per year, the impact on an operational suite is very minor, but one is sure to detect and improve the most extreme ones.

The restarted model run should be initialized, to get a balanced state after the spatial interpolation of the fields to the resolution of the LAM. The ALADIN model is initialized by a digital-filtering initialization (DFI), a technique that was introduced by Lynch (1990), which consists of applying a digital low-pass temporal filter on the initial state of the forecast to remove all modes with frequencies that are assumed to lie within the time spectrum of the gravity waves, while also assuming that the remaining low time frequencies belong to the meteorologically relevant part of this state.

Termonia (2008, hereinafter T08) identified a Doppler effect in the temporal spectrum of the ALADIN forecast of the Lothar storm that shifts the frequencies of the meteorologically relevant modes. This Doppler effect occurs when a storm grows on top of a fast-propagating large-scale flow. The Doppler shift is proportional to its propagation speed and for sufficiently high velocity this may shift meteorologically relevant frequencies into the 
TABLE 1. Boundary error cases within the period from 21 Feb 2006 to 21 Jun 2008 with their date, boundary sampling error detection time, HFSP value, their type, and corresponding BER time. There were two times two subsequent boundary sampling error detection times indicated by the braces. The mslp of these cases are shown in the figures indicated in the last column.

\begin{tabular}{|c|c|c|c|c|c|}
\hline Date & $\begin{array}{l}\text { Boundary sampling error } \\
\text { detection time }(\mathrm{h})\end{array}$ & HFSP value & Type & BER time (h) & Figure \\
\hline 24 Nov 2006 & $\left\{\begin{array}{l}+24 \\
+27\end{array}\right.$ & $\left.\begin{array}{l}0.0044 \\
0.0058\end{array}\right\}$ & Incoming & +27 & $\begin{array}{l}7 \mathrm{a} \\
7 \mathrm{~b}\end{array}$ \\
\hline 25 Nov 2006 & +6 & 0.0033 & Incoming & +9 & $7 \mathrm{c}$ \\
\hline 1 Dec 2006 & +21 & 0.0033 & Corner (NE) & +24 & $7 \mathrm{~d}$ \\
\hline 31 Dec 2006 & $\left\{\begin{array}{l}+15 \\
+18\end{array}\right.$ & $\left.\begin{array}{l}0.0047 \\
0.0040\end{array}\right\}$ & Tangent & +18 & $\begin{array}{l}8 \mathrm{a} \\
8 \mathrm{~b}\end{array}$ \\
\hline 18 Jan 2007 & +24 & 0.0045 & Outgoing & +30 & $8 \mathrm{c}$ \\
\hline 8 Dec 2007 & +30 & 0.0041 & Incoming & +33 & $8 \mathrm{~d}$ \\
\hline
\end{tabular}

frequency domain that is supposed to be exclusively occupied by the gravity waves. This was particularly the case for the Lothar storm studied in T03, T04, and T08, propagating with a velocity of about $100 \mathrm{~km} \mathrm{~h}^{-1}$. As a consequence, a DFI similar to that used in Lynch and Huang (1992) with a cutoff period of $3 \mathrm{~h}$ removed about 6-7 hPa from the low of this storm. It was then shown in T08 that this can be avoided by introducing a scaleselective DFI (SSDFI).

So, the aim here is to apply a BER to avoid an error of about $10 \mathrm{hPa}$ caused by the LBC temporal resolution problem. However, if we then initialize the initial state of this restarted forecast by means of a standard DFI, we may, ironically, lose again about $10 \mathrm{hPa}$ due to this Doppler shift. Therefore, the BERs are initialized by an SSDFI, with the cutoff period of the filter ${ }^{5}$ taken to be $1 \mathrm{~h}$ and the cutoff wavenumber $\kappa_{c}=2 \pi \times 10^{-5} \mathrm{~m}^{-1}$, corresponding to a wavelength $L_{c}=100 \mathrm{~km}$ (see T08 for details concerning $\kappa_{c}$ and $L_{c}$ ). This filter will be denoted as $\operatorname{SSDFI}_{1 \mathrm{~h}}$.

The storms detected in Table 1 did not propagate as fast as the Lothar storm. So, there is not a great need for an SSDFI in the examples presented here. However, the propagation speed cannot be anticipated. So a decision was made to always use SSDFI instead of DFI in order to be properly prepared for the next storm propagating with a very high velocity (e.g., $\sim 100 \mathrm{~km} \mathrm{~h}^{-1}$, as the past Lothar storm).

Figure 9 shows the effects of the SSDFI at 33-h forecast range for the 0000 UTC 8 December 2007 run (Fig. 8d) on the balance ratio (Br) introduced by Lynch and Huang (1992) (see also T08 within the context of SSDFI),

\footnotetext{
${ }^{5}$ The tests presented here were carried out with a DolphChebyshev filter applied with stop-band edge of $1 \mathrm{~h}$, time span of $0.833 \mathrm{~h}$, and ripple ratio $r=0.05$; see Lynch (1997) for an explanation of these parameters.
}

$$
\mathrm{Br}=100 \frac{\sum_{I J}\left|\sum_{L} \boldsymbol{\nabla} \cdot \Delta_{p_{L}} \mathbf{V}_{I J L}\right|}{\sum_{I J} \sum_{L}\left|\nabla \cdot \Delta_{p_{L}} \mathbf{V}_{I J L}\right|},
$$

giving a measure of the balance of the atmospheric state, where $\mathrm{V}$ is the horizontal wind vector labeled with gridpoint indices $I, J$, and $L$ for the vertical sigma level. Also, $\Delta_{P L}$ denotes the pressure difference between the two half-levels above and below level $L$. Indeed, the SSDFI reduces $\mathrm{Br}$ from 17.4 to 10.6. The effect is, albeit less, comparable to the one that was obtained for the Lothar storm in T08.

As can be seen from Fig. 9, the effect of the SSDFI is short. For this particular case, one might even consider not initializing at all. Nevertheless, it has been checked (not shown) that SSDFI does not substantially diminish the low of the storm compared to employing no initialization, but it still provides some initialization in the case of a Lothar-type storm propagating into the domain.

For the detected boundary sampling error in the 0000 UTC 24 November 2006 run (Figs. 7a and 7b), we performed a BER at 27 -h forecast range. The mslp at those times are displayed in Fig. 10. The storm enters the domain at the west side. Figure 10a shows the mslp at 27-h forecast range. It can be seen in Fig. 10a that, as a direct consequence of the interpolation error of the LBC data, the low of this storm $(971.1 \mathrm{hPa})$ was $2.7 \mathrm{hPa}$ less deep in the LAM than that in the coupling model $^{6}(968.4 \mathrm{hPa})$ displayed in Fig. 10c. Figure 10e shows the result of the BER after applying the $\mathrm{SSDFI}_{1 \mathrm{~h}}$. It can be seen, from comparing this BER with the $27-h$ forecast range of the run in Fig. 10a, that the storm is $3 \mathrm{hPa}$ deeper at the restart and that the gradient of the

\footnotetext{
${ }^{6}$ This coupling model was run on the larger domain shown in Fig. 1. Figure 10c shows its mean sea level pressure interpolated on the smaller domain of the coupled LAM without initialization.
} 

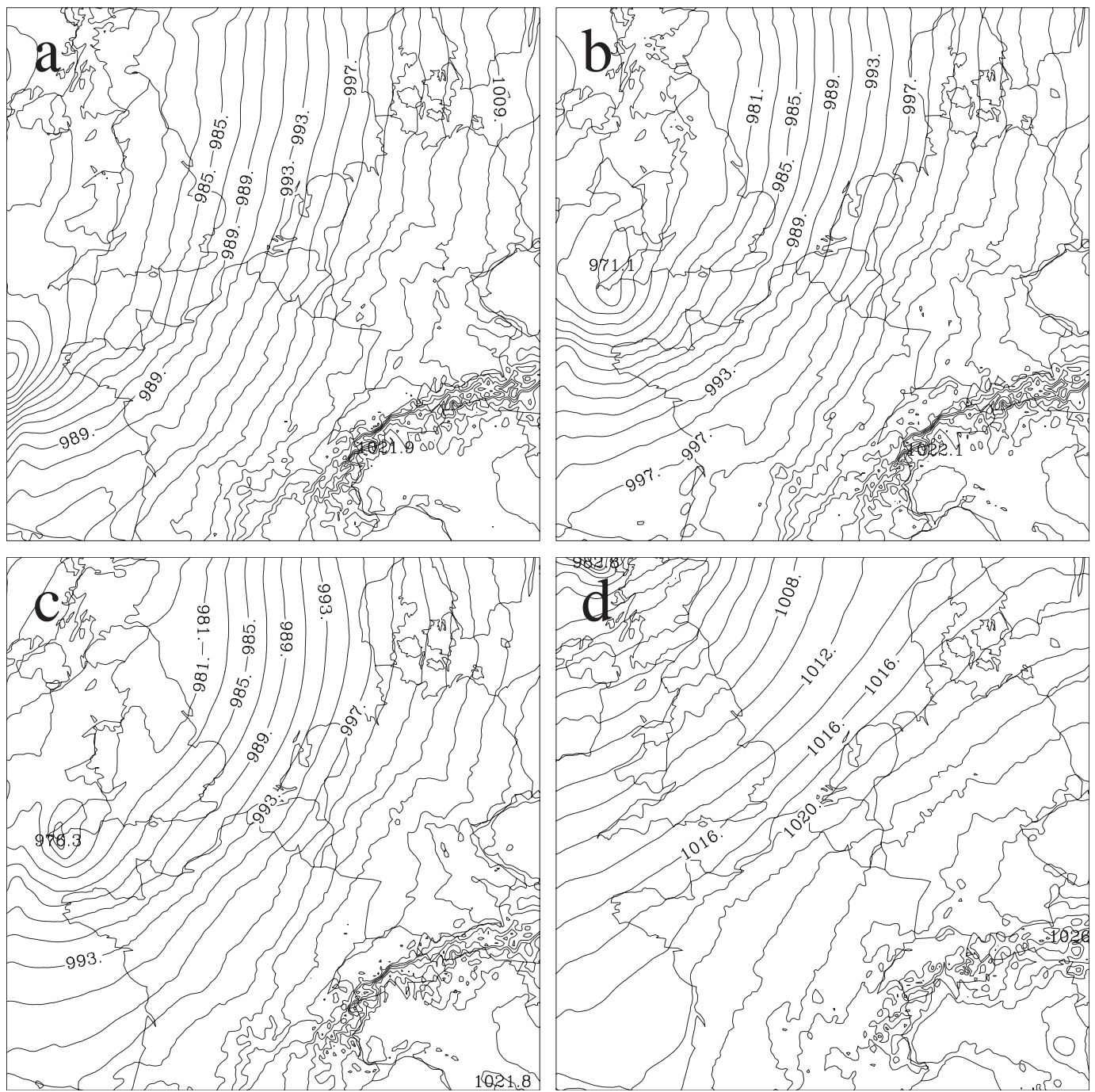

FIG. 7. The mslp during the boundary sampling error detections in the 0000 UTC runs: (a) 24-h forecast range of the run on 24 Nov 2006, (b) 27-h forecast range of the run on 24 Nov 2006, (c) 6-h forecast range of the run on 25 Nov 2006, and (d) 21-h forecast range of the run on 1 Dec 2006.

mslp around it is much stronger. The strength of the storm was also substantially improved after the restart time. For instance, 3 h later, Figs. 10b, 10d, and 10f show the situation of the 30 -h forecast range of the LAM, the mslp of the coupling model, and the 3 -h forecast range of the BER. The low is $1 \mathrm{hPa}$ deeper, but the gradient is still much stronger for the run after the BER. For the same storm in the 0000 UTC 25 December 2006 run in Table 1, the same conclusions were drawn (not shown).

In the 0000 UTC 8 December 2007 run (Fig. 8d), we had a boundary sampling error detection at 30-h forecast range. The mslp at 33-h forecast range is given in Fig. 11a. Figure 11c shows the mslp of the coupling model, and Fig. 11e shows the corresponding mslp at the restart of the BER. In comparison with the previous case, the im- provement in the mslp gradient is less significant. However, the depth of the low has been improved by $5.5 \mathrm{hPa}$. Figures 11b, 11d, and 11f show the situation 3 h later. By then, the storm is already weakening but the difference in pressure is still $4.4 \mathrm{hPa}$. Note that, because of the application of the $\mathrm{SSDFI}_{1 \mathrm{~h}}$, the mslp of the BER in Fig. 11f is better adjusted to the orography of the Alps than is the coarser one in the coupling model in Fig. 11d.

The tests presented in this section show that (i) it is feasible to detect the severest boundary sampling errors caused by the LBC temporal resolution problem, (ii) this is feasible in an operational forecast suite, and (iii) the problem of the initialization mentioned in T08 can be avoided in the restarts by relying on a scale-selective digital-filtering initialization. Performing BERs in the 

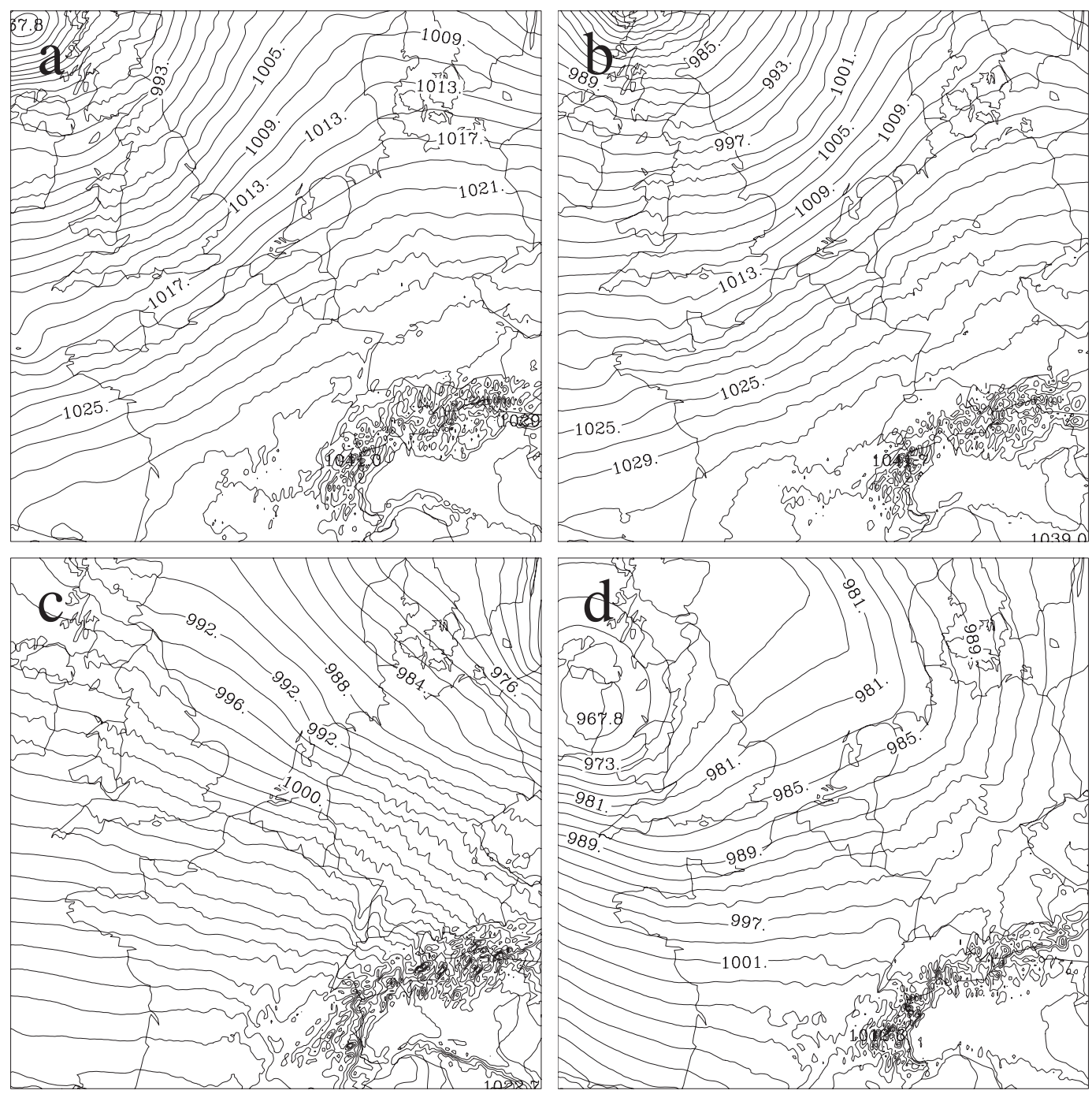

FIG. 8. The mslp during the boundary sampling error detections in the 0000 UTC based runs: (a) 15-h forecast range of the run on 31 Dec 2006, (b) 18-h forecast range of the run on 31 Dec 2006, (c) 24-h forecast range of the run on 18 Jan 2007, and (d) 30-h forecast range of the run on 8 Dec 2007.

tangent and outgoing cases did not degrade the forecasts. This shows that an automated BER procedure (which, e.g., cannot distinguish incoming from outgoing cases) will not harm the forecast quality of the storms in these cases.

\section{Discussion}

The subject of this paper was the LBC temporal resolution problem. The first aim of this paper was to replace the guesswork in the operational practice by a more scientifically justified approach. We recommend that the choice of the coupling update frequency should be based on the curves presented in Fig. 3. Such curves allow the users of the LAM to choose the required coupling update frequency that guarantees that the difference between the original and interpolated surface pressures will never exceed a predetermined maximal error. For instance, considering Fig. 3 we see that, to have a guarantee that the error between the interpolated and original values never exceeds $1 \mathrm{hPa}$, a coupling update of $20 \mathrm{~min}$ is required, and this is practically the time step of the coupling model (ARPEGE) in this case. The same curve was also computed at the same grid point for the 48-h run on 18 December 1999, also indicated as a dashed line in Fig. 3. This is a normal anticyclonic winter situation without a storm, taken within the period studied in T04 (in particular, see Fig. 8 of that paper). It can be seen from Fig. 3 that, in that case, the commonly used 3-h time interval employed by the 
operational ALADIN models suffices to guarantee a maximal error of $1 \mathrm{hPa}$.

Computing the curves in Fig. 3 is easy and it is thus easy to repeat the analysis for future storms, if they turn out to be more severe than the Lothar storm, to adjust the current ad hoc choice.

If the required temporal resolution is too high to use in practice, this paper proposes a nesting strategy based on boundary error restarts to improve the forecasts of the strength of the severest storms encountered in the operational suite. It shows how the monitoring of the coupling update frequency proposed in T04 can be used to determine the restart time in an operational forecast suite, before the coupling data are used in the coupled LAM. To avoid fast-propagating storms becoming corrupted by the Doppler effects identified in T08, these restarts must be initialized by a scale-selective digitalfiltering initialization.

Of course, after a restart the small-scale part of the interrupted forecast is lost. In the cases of the storm forecasts detected in the present paper, the improvement of the low clearly outweighs this, as can be seen in Figs. 10 and 11. However, this should be studied in more detail and whether spectral techniques, similar as the ones introduced by Waldron et al. (1996) and von Storch et al. (2000), could be used to nudge the small scales to the ones of the interrupted forecast at the time of the restart should be investigated. But this lies beyond the scope of the present paper. The obtained improvements are sufficient to justify further research along these lines.

Over 2 yr of operational data have been investigated. Based on a reasonable choice of the threshold value of the high-pass-filtered surface pressure field within a frame that covers the Davies relaxation zone, eight boundary sampling errors were detected, two of them corresponding to a storm that was already detected $3 \mathrm{~h}$ earlier. There were three incoming cases: one tangent, one outgoing, and one corner case. In practice, this implies that one should expect only a few of these BERs per year in the operational suite of the LAM, including the most extreme storms. For the studied cases, it was indeed observed that using a too long coupling update interval makes the depressions less deep after they have been coupled by the interpolated data on the lateral boundaries. In the detected cases, it was then shown how this is corrected for by the BERs.

More BERs could be generated by specifying a lower value of $\mu_{c}$ for the operational suite, but these extra BERs would correct weaker storms and smaller boundary errors. In the cases with a BER, the restarted forecast could either replace the model run or it could be carried out as an additional run to be provided to the forecaster, either as a better estimation of the strength of the storm or as an

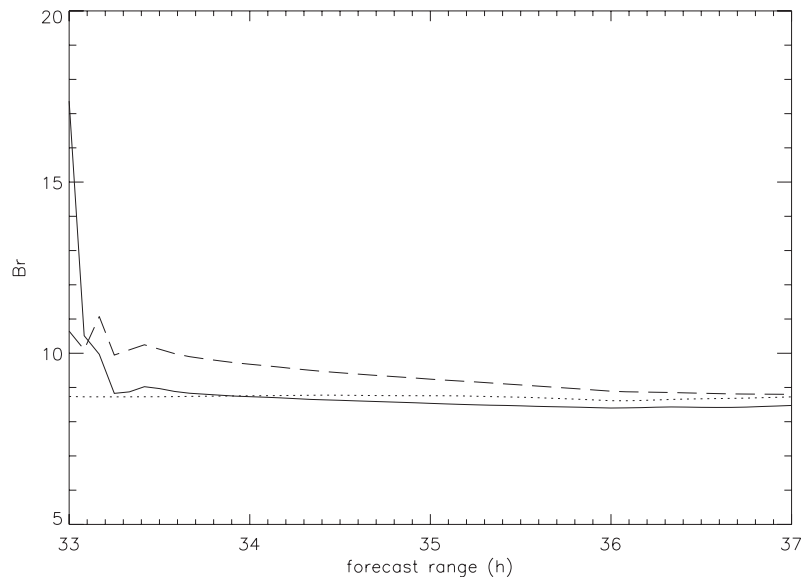

FIG. 9. The Br after the 33-h forecast range of the 0000 UTC 8 Dec 2007 forecast (dots) and the BER after the 33-h forecast range with SSDFI (dashes) and no initialization (solid line).

estimate of the error induced by the LBC temporal resolution problem. Although the effect is less than for the Lothar storm forecast studied in T04, the improvements shown in the detected cases are very substantial.

The proposed nesting strategy does not contradict the guideline of Warner et al. (1997) to have a sufficiently large domain to keep the lateral boundaries far enough from the region of interest such that the corrupted signal will not contaminate it. The present strategy complements this approach in two ways: (i) by avoiding the use of corrupted data at the boundaries and (ii) by the restarts guaranteeing that the feature of interest is inside the domain from the beginning of the forecast.

The other nesting strategy mentioned in T04, of increasing the temporal resolution in cases of boundary sampling error detections, has not been investigated. In this case the run of the LAM is not interrupted but the coupling update frequency would be adapted during the passage of the feature through the coupling domain. This does not solve the problem of the strong (dynamical) forcing at the boundaries and may, thus, still induce a coupling error that will propagate to the interior domain. This also has the disadvantage that the forecast of the LAM can be delayed due to the transmission and I/O of the extra coupling data.

In operational suites where transmission costs are not an issue, coupling with the frequency corresponding to the time step of the coupling model should be seriously considered. But it should be noted that in those cases, the coupling model may be slowed considerably in order to produce these outputs. However, even in that case, restarts may turn out to be useful. For instance, recent work of A. McDonald (2006, personal communication) showed that there may exist critical levels of gravity 

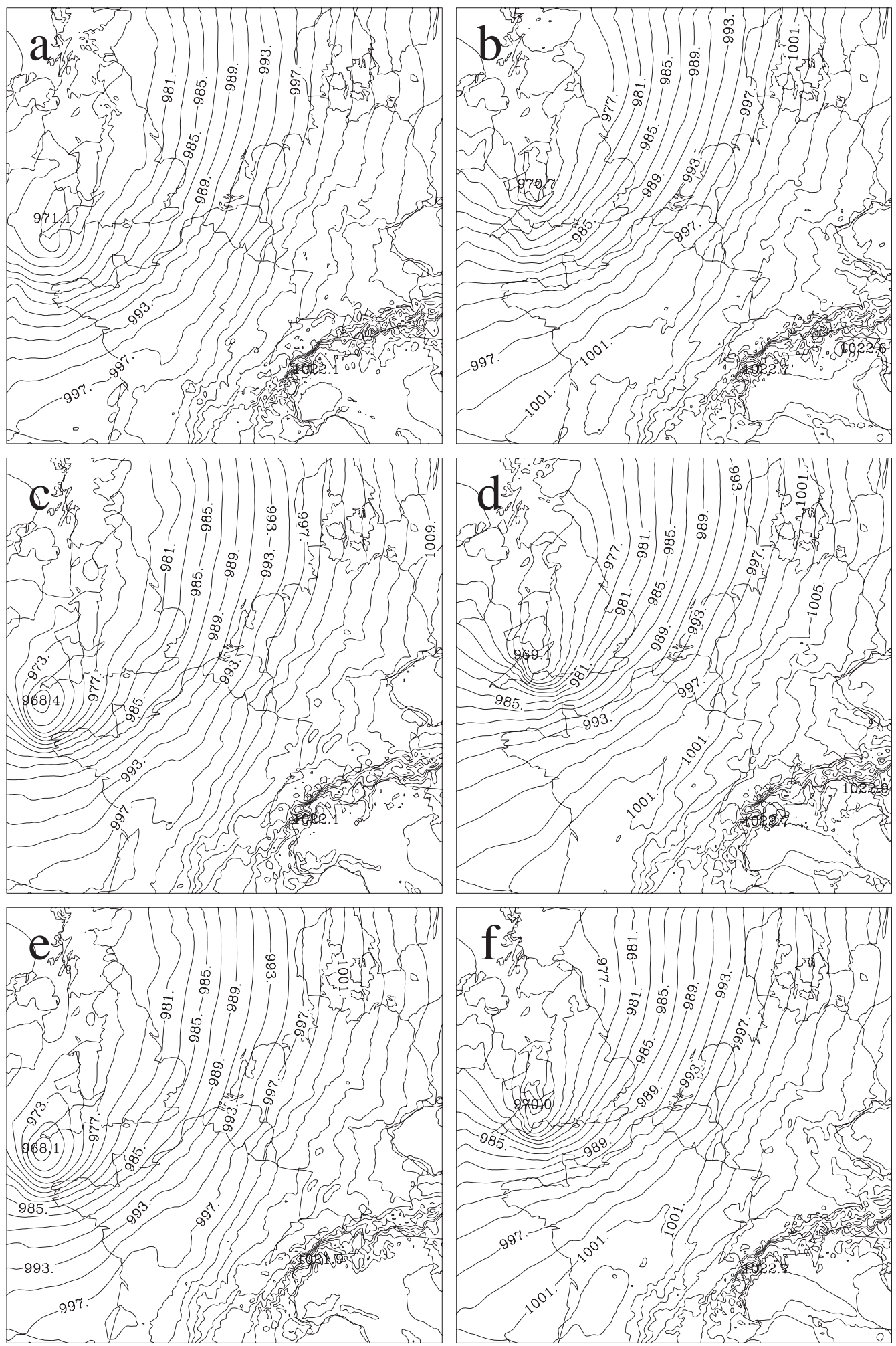

FIG. 10. The mslp of the (a) 27-h forecast range (low $971.1 \mathrm{hPa}$ ) and (b) 30-h forecast range $(970.7 \mathrm{hPa})$ of the 0000 UTC 24 Nov 2006 forecast. Also shown is the content of the coupling model (ALADIN Météo-France) at (c) 0300 UTC $(968.4 \mathrm{hPa})$ and (d) $0600 \mathrm{UTC} 25 \mathrm{Nov} 2006(969.1 \mathrm{hPa})$, and the corresponding output of the BER run performed at the 27-h forecast range at (e) $0300 \mathrm{UTC}(968.1 \mathrm{hPa})$ and (f) $0600 \mathrm{UTC} 25 \mathrm{Nov} 2006$ (970.0 hPa). 

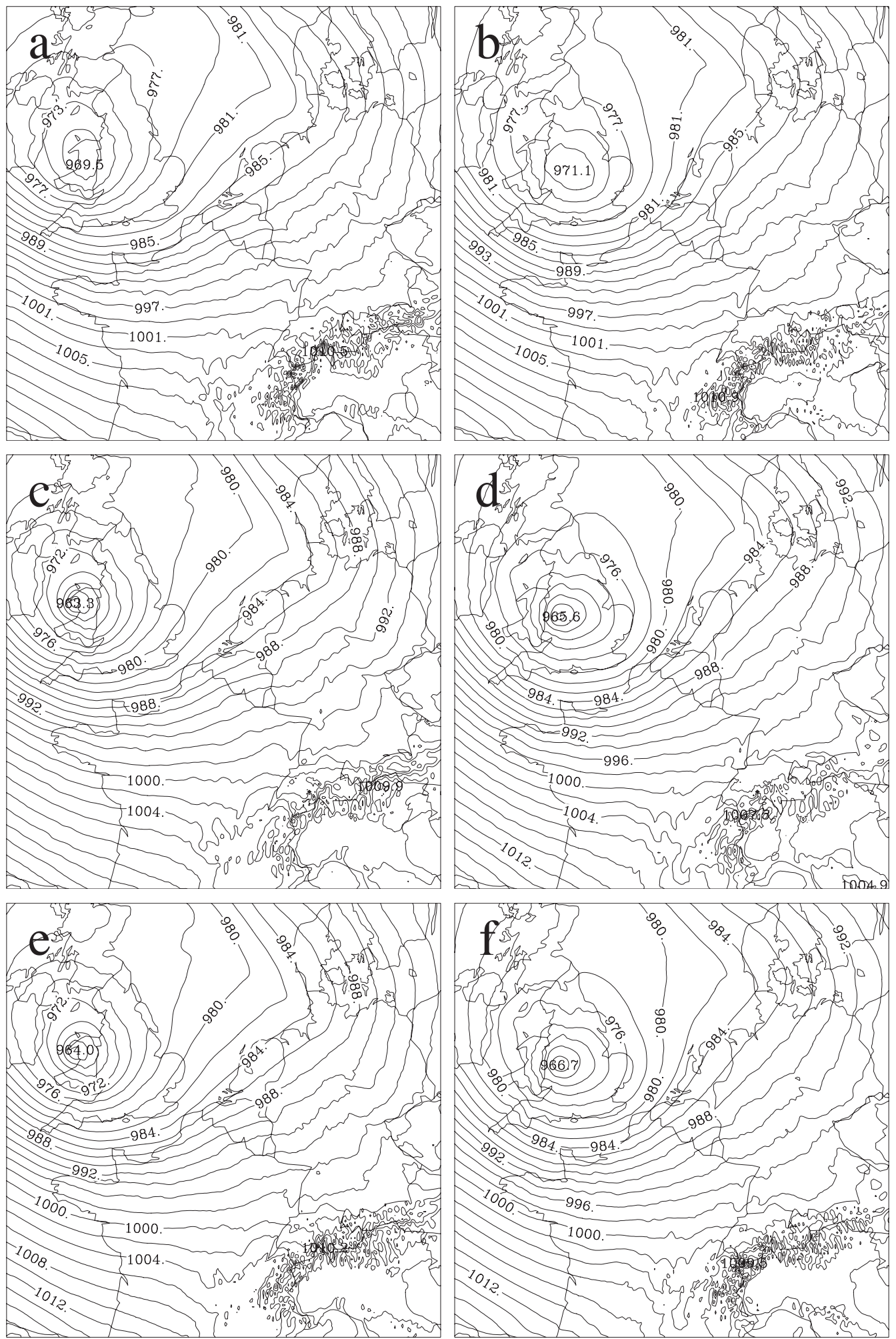

FIG. 11. The mslp of the (a) 33-h forecast range (low $969.5 \mathrm{hPa}$ ) and (b) 36-h forecast range $(971.1 \mathrm{hPa}$ ) of the 0000 UTC 8 Dec 2007 forecast on. Also shown is the content of the coupling model (ALADIN Météo-France) at (c) 0900 UTC (963.3 hPa) and (d) 1200 UTC 8 Dec $2007(965.6 \mathrm{hPa})$, and the corresponding times of the BER performed at the 33-h forecast range at (e) $0900 \mathrm{UTC}(964.0 \mathrm{hPa})$ and (f) $1200 \mathrm{UTC} 8 \mathrm{Dec} 2007$ (966.7 hPa). 
waves to be imposed in well-posed formulations beyond which they lose their reliability. A monitoring strategy with restarts could also turn out to be the solution in that case.

Acknowledgments. This paper was very much improved by many pertinent comments and questions raised by the two anonymous reviewers.

\section{REFERENCES}

ALADIN International Team, 1997: The ALADIN project: Mesoscale modelling seen as a basic tool for weather forecasting and atmospheric research. WMO Bull., 46, 317-324.

Baumhefner, D. P., and D. J. Perkey, 1982: Evaluation of lateral boundary errors in a limited-domain model. Tellus, 34, 409-428.

Davies, H. C., 1976: A lateral boundary formulation for multilevel prediction models. Quart. J. Roy. Meteor. Soc., 102, 405-418.

Errico, R. M., 1985: Spectra computed from a limited area grid. Mon. Wea. Rev., 113, 1554-1562.

Haugen, J. E., and B. Machenhauer, 1993: A spectral limited-area model formulation with time-dependent boundary conditions applied to the shallow-water equations. Mon. Wea. Rev., 121, 2618-2630.

Lynch, P., 1990: Initialization using a digital filter. Research Activities in Atmospheric and Ocean Modeling, CAS/JSC Working Group on Numerical Experimentation Rep. 14, WMO Secretariat, Geneva, Switzerland, 1.5-1.6.

—, 1997: The Dolph-Chebyshev window: A simple optimal filter. Mon. Wea. Rev., 125, 655-660.

, and X.-Y. Huang, 1992: Initialization of the HIRLAM model using a digital filter. Mon. Wea. Rev., 120, 1019-1034.
McDonald, A., 2005: Transparent lateral boundary conditions for baroclinic waves: A study of two elementary systems of equations. Tellus, 57A, 171-182.

_ 2006: Transparent lateral boundary conditions for baroclinic waves. II. Introducing potential vorticity waves. Tellus, $\mathbf{5 8 \mathbf { A }}$, 210-220.

Termonia, P., 2003: Monitoring and improving the temporal interpolation of lateral-boundary coupling data for limited-area models. Mon. Wea. Rev., 131, 2450-2463.

_ 2004: Monitoring the coupling-update frequency of a limitedarea model by means of a recursive digital filter. Mon. Wea. Rev., 132, 2130-2141.

_ 2008: Scale-selective digital filtering initialization. Mon. Wea. Rev., 136, 5246-5255.

— conditions from the dynamic core in semi-implicit semiLagrangian models. Tellus, 60A, 632-648.

Vannitsem, S., and F. Chomé, 2005: One-way nested regional climate simulations and domain size. J. Climate, 18, 229-233.

Voitus, F., P. Termonia, and P. Bénard, 2009: Well-posed lateral boundary conditions for spectral semi-implicit semiLagrangian schemes: Tests in a one-dimensional model. Mon Wea. Rev., 137, 315-330.

von Storch, H., H. Langenberg, and F. Feser, 2000: A spectral nudging technique for dynamical downscaling purposes. Mon. Wea. Rev., 128, 3664-3673.

Waldron, K. M., J. Peagle, and J. D. Horel, 1996: Sensitivity of a spectrally filtered and nudged limited area model to outer model options. Mon. Wea. Rev., 124, 529-547.

Warner, T. T., R. A. Peterson, and R. E. Treadon, 1997: A tutorial on lateral boundary conditions as a basic and potentially serious limitation to regional numerical weather prediction. Bull. Amer. Meteor. Soc., 78, 2599-2617.

Wernli, H., S. Dirren, M. A. Liniger, and M. Zillig, 2002: Dynamical aspects of the life cycle of the winter storm 'Lothar' (2426 December 1999). Quart. J. Roy. Meteor. Soc., 128, 405-429. 